\title{
Support Vector Machines for Young and Older Gait Classification using Inertial Sensor Kinematics at Minimum Toe Clearance
}

\author{
Braveena K. \\ Santhiranayagam \\ Institute of Sport, Exercise \\ Science and Active Living \\ College of Sport and Exercise \\ Science \\ Victoria University, \\ Melbourne, Australia \\ braveena.santhiranayagam \\ @live.vu.edu.au
}

\author{
Daniel T. H. Lai \\ College of Engineering and \\ Science \\ Victoria University \\ Melbourne, Australia \\ daniel.lai@vu.edu.au
}

\author{
Rezaul K. Begg \\ Institute of Sport, Exercise \\ Science and Active Living \\ College of Sport and Exercise \\ Science \\ Victoria University, \\ Melbourne. Australia \\ rezaul.begg@vu.edu.au
}

\begin{abstract}
The present study investigates the inertial sensor kinematics obtained at a critical toe-control event, Minimum Toe Clearance (MTC), to classify different age groups. Fourteen young and fourteen older adults performed treadmill walking at their preferred walking speed, wearing a shoe-mount inertial sensor unit measuring tri-axial acceleration and triaxial angular velocities. Three dimensional (3D) positiontime data was obtained using high accurate motion capture system. MTC timing within a gait cycle $\left(M T C_{\text {Time }}\right)$, calculated using 3D motion capture data, was used to extract inertial sensor kinematics at MTC event. Mean and standard deviation of three inertial sensor acceleration features and three angular velocity features were compared between young and older individuals using t-tests. Young adults' mean anterior-posterior acceleration was greater than older adults $(\mathrm{p}=0.002)$. Further, standard deviations (SD) of all three accelerations and angular velocity about medio-lateral axis were greater in Older adults. The inertial sensor kinematics obtained at $M T C_{\text {Time }}$ were able to classify young and older adults gait with $91.2 \%$ accuracy using a Support Vector Machine (SVM) classifier. The findings of the present study suggest that by employing SVM techniques, a portable inertial sensor system could be used to identify gait degeneration due to ageing and has the potential for wider applications in gait identification for falls-risk minimization.
\end{abstract}

\section{Keywords}

inertial sensor, accelerometer, gyroscope, minimum toe clearance, support vector machine

\section{INTRODUCTION}

As people age, their risk of falling increases and the consequences of a fall are more serious. Worldwide the population aged more than 60 was estimated to be 688 million in 2006 but is projected to grow to almost two billion by 2050 [9]. From a biomechanical perspective, falls during locomotion result from different destabilizing events such as tripping, slipping and loss of balance. Of these falls-related biomechanical events, tripping accounts for more than $50 \%$ of falls [13] and a high association between tripping frequency and falling was reported in community-dwelling older adults [10].

Tripping results directly from unsuccessful toe-ground clearance, primarily during the swing phase of a gait cycle. Previous research, therefore, focused on examining the lower limb swing-phase trajectory control, represented by a biomechanical event during the mid-swing phase of the gait cycle, Minimum Toe Clearance (MTC) of young and older populations to understand the ageing effects $[2,6,8]$. Low toeground clearance at MTC $\left(M T C_{\text {Height }} \sim 10-20 \mathrm{~mm}\right)$ in addition to high foot velocity $(\sim 4.60 \mathrm{~m} / \mathrm{s})$ and a single-foot base of support poses a significant hazard to locomotion. Failure to adequately compensate surface height variability by adjusting clearance at MTC, increases tripping risk. Older adults maintained their mean $M T C_{H e i g h t}$ similar to young individuals' $[2,8]$ but their $M T C_{\text {Height }}$ dispersion, characterized by either the standard deviation (SD) or inter quartile range $(\mathrm{IQR})$ are greater than the young. This greater stride-to-stride variability in $M T C_{\text {Height }}$ observed in older adults, increases the chances of toe-ground contact while walking $[1,2,8]$.

Automated recognition of gait degeneration is the first step in developing different falls-risk minimization applications in the older, such as early identification of risky gaits, active intervention to modify the risky gaits, and monitoring the progress of treatment outcomes. The present study investigates accelerometer and angular velocity measurements obtained from a portable, compact and light-weight inertial sensor system to automatically classify young and older adults' gait using Support Vector Machines (SVM). MTC, as a cyclic event, has two physical characteristics, $M T C_{H e i g h t}$, 


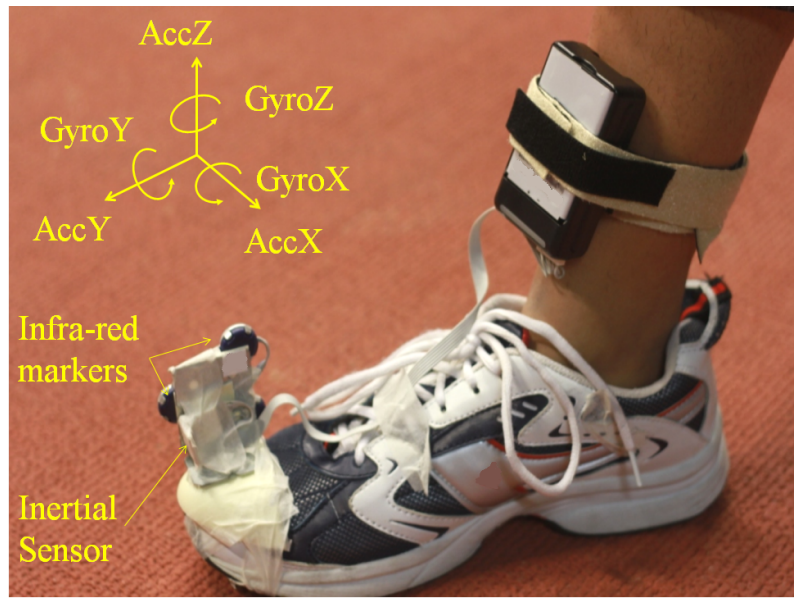

Figure 1: Rigid body marker set up and inertial sensor attached to distal end of the shoe. The axes of inertial sensor unit were marked in yellow. The battery pack and data transmission unit was attached to the shank.

as discussed above and timing of MTC event, i.e., time of MTC event occurrence within the gait cycle $\left(M T C_{\text {Time }}\right)$. Mills et al. [8] used $M T C_{\text {Time }}$ to analyse the lower limb joint angles at the MTC event to further understand swing phase biomechanics. Mills et al. [8] reported that older adults displayed less stance hip extension, greater swing hip flexion and less stance hip adduction at $M T C_{\text {Time }}$ than the young. Further, greater MTC variability observed in older was mostly correlated with swing stance ankle adductionabduction variability, whereas, for young MTC variability was mostly correlated with ankle plantar-dorsiflexion variability [8]. While it is reasonable to suggest that kinematics obtained from inertial sensor signals at $M T C_{\text {Time }}$, would indicate greater variability in older adults, no previous study has analysed inertial sensor signals at $M T C_{T i m e}$.

\section{EXPERIMENTAL METHODS}

The experiment was conducted in the Victoria University Biomechanics Laboratory, Melbourne, Australia. Fourteen young healthy adults and fourteen older adults aged $65+$ with the ability to perform everyday walking for 30 minutes without a walking aid and having no orthopaedic, respiratory and cardiac conditions were recruited. Older adults also underwent following screening tests: (i) timed up and go $(<13.5$ secs [5], (ii) visual acuity $(>6 / 12)$ and (iii) contrast sensitivity (Melbourne edge test $>6 / 15$ [7]. A wireless foot-mount sensor module was employed with 6 degrees of freedom (DOF) consisting of an accelerometer - ADXL345 and a gyroscope- ITG3200 to measure the distal foot linear accelerations and angular velocities (Figure $1 \mathrm{~A})$. The ultra low-powered tri-axis accelerometer had a $\pm 16 \mathrm{~g}$ capacity in full-scale, a sensitivity of $31.2 \mathrm{LSB} / \mathrm{g}$ and a maximum $3200 \mathrm{~Hz}$ bandwidth. The ITG3200 16 bit digital gyroscope had a sensitivity of $14.375 \mathrm{LSBs} / \mathrm{sec}$ and a full-scale range of $\pm 2000^{\circ} / \mathrm{sec}$. The sensing unit was powered by a Sony Ericsson BST-41 Li-Polymer rechargeable Battery with an energy capacity of $1500 \mathrm{mAh}$ that could transmit $100 \mathrm{~Hz}$ data wirelessly for approximately 11 hours. The embedded onboard system was implemented on a Freescale Semicon-

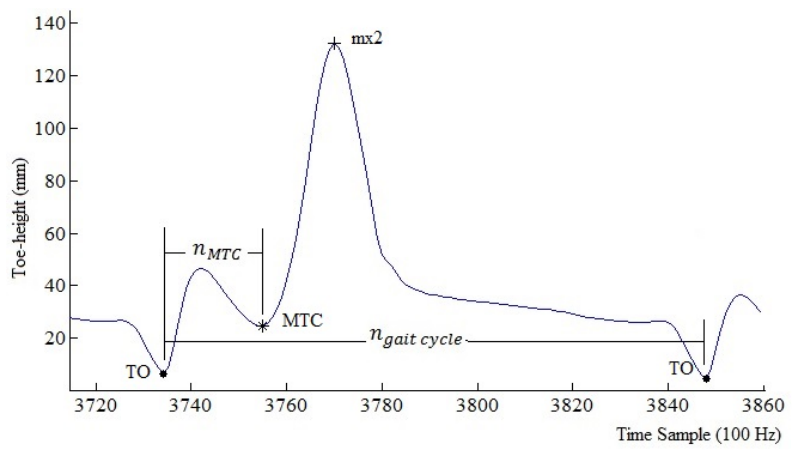

Figure 2: Toe-height over time for a typical gait cycle with an MTC event. At toe-off the toe breaks the contact with the ground and enters into the swing phase and at $\mathrm{mx} 2$ toe reaches the maximum vertical clearance. $M T C_{\text {Time }}$ is calculated using $n_{M T C}$, number of samples from TO event to MTC and $n_{\text {gaitcycle }}$, number of total samples within the gait cycle, defined from one TO to the consecutive TO event.

ductor MCU (8-bit MC9S08SH8) and Bluetooth 2.0/EDR communications were used to (Sena ESD200/210) transfer the sensor data to a computer. A Matlab GUI was written to communicate and store data from the sensor units.

\subsection{Data Collection}

All participants completed informed consent procedures approved by the Victoria University Research Ethics Committee. A safety harness was worn while walking on the motorized treadmill. A rigid body comprising 3 infra-red emitting diodes was attached to the distal end of the right shoe and an imaginary marker was digitized at the lowest distal extremity of the shoe to represent the toe with respect to the rigid body (Figure 1). The three-dimensional (3D) coordinates of the markers were tracked relative to a three dimensional labbased reference system. The horizontal plane of the global coordinate system was the treadmill deck surface, with the anterior-posterior axis directed in line with the treadmill belt motion. Custom built shoe-mount sensor unit was also attached to the distal end of the right shoe and a laptop was used to collect wirelessly transferred inertial sensor signals.

Participants' preferred walking speed (PWS) on the treadmill was determined by first increasing the treadmill speed until the participant reported the speed to be uncomfortably fast (fast limit). It was then decreased until reported to be uncomfortably slow (slow limit). The mean of three fast and three slow limits was considered as PWS. When required, participants were given 10-15 minutes familiarization before determining PWS. Participants walked at PWS for 5 mins. In first and last $30 \mathrm{~s}$ of the trial, participants did not walk, and the standing duration was used to sync 3D motion capture and foot-mount sensor data (Figure 3).

\subsubsection{D motion capture data}

Position-time data from the Optotrak 3D motion capture system was exported to Visual3D (C-motion, Canada) analysis software and the raw data were first interpolated to compensate any occluded signals using a window of up to 10 frames (0.1s). A 4th order zero-lag Butterworth Filter 

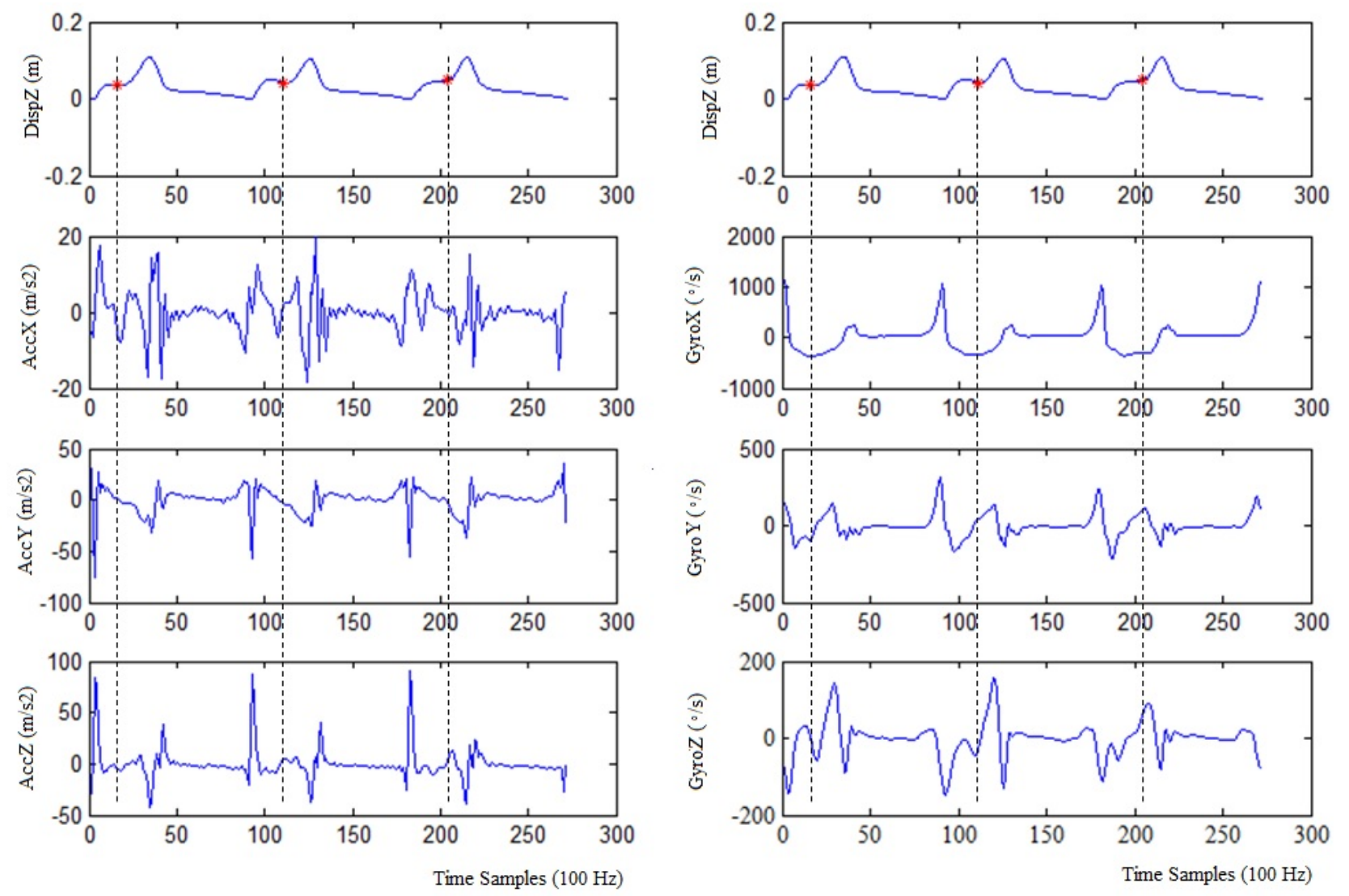

Figure 3: Time synchronized 3D motion capture vertical toe-displacement signals and corresponding tri-axial accelerometers and tri-axial gyroscopes obtained from a typical young participant for 3 complete gait cycles. * denotes MTC event in 3D motion capture data.

with a cut-off frequency of $12 \mathrm{~Hz}$ was then applied to toe displacement data. Conditioned data were saved as text files for further processed using in-house developed MATLAB v7.2 scripts (The Mathworks, Natick, MA, USA).

MTC is found in the characteristic vertical displacement trough between Toe-off (TO) and $\mathrm{mx} 2$ (Figure3). Once MTC was identified within the 3D motion capture data, $M T C_{\text {Time }}$ was calculated as a percentage of total number of samples within a gait cycle using the formula:

$$
M T C_{\text {Time }}=\frac{n_{M T C}}{n_{\text {gaitcycle }}} * 100
$$

where $n_{M T C}$ is number of samples from a toe-off (TO) event to MTC, and $n_{\text {gaitcycle }}$ is number of total samples within the gait cycle, defined from one toe-off to the consecutive toe-off event (Figure 2).

\subsubsection{Inertial Sensor Signal Processing}

Three dimensional acceleration measurements obtained from the IMU were respectively foot acceleration along the mediolateral axis (AccX), anterior-posterior axis (AccY) and longitudinal axis (AccZ) (Figure 3). Foot angular velocity about the medio-lateral axis (AngVelX), anterior-posterior axis
(AngVelY) and longitudinal axis (AngVelZ) were measured using gyroscope. Both accelerometers and gyroscopes signals were high-pass filtered forward and reverse using a 2nd order Butterworth filter (cut-off frequency $1 \mathrm{~Hz}[6]$ ) to ensure zero phase shift and to remove any sensor drift. Voltage outputs $(\mathrm{V})$ of the IMU sensor were converted to SI (Standard-International) units using sensitivity scaling factor:

- Accelerometer (acc): $9.812 * V_{a c c} * 31.2 / 1000$

- Gyroscope (gyro): $V_{\text {gyro }} / 14.375$

Toe-off event in the inertial sensor signals were represented by the maximum medio-lateral toe angular velocity [11]. Inertial sensor signals and 3D positional data were then synchronised using toe-off events. Calculated $M T C_{\text {Time }}$ was then used to extract inertial sensor kinematics. In gait cycles which did not show an MTC event, participant-specific mean $M T C_{\text {Time }}$, calculated over multiple gait cycles which showed an MTC event, was used to extract inertial signal kinematics for further analysis.

\subsection{Data Analysis}


Mean and standard deviation (SD) were calculated for each inertial signal kinematics (3 accelerometer signals and 3 gyroscope signals) extracted at $M T C_{\text {Time }}$ for both Young and Older separately. Six mean and six SD values between Young and Older were statistically compared using t-test with significance set at .05 .

\subsubsection{Support Vector Machine (SVM)}

Support vector machines (SVMs) $[14,15,17]$ are binary classifiers based on Vapnik's structural risk minimisation theories [17] which achieves a trade-off between empirical risk (training set error) minimisation (ERM) and regularisation to avoid the problem of overfitting. Moreover, by using the kernel trick the SVM is able to overcome the so-called curse of dimensionality $[3,4,12,16]$.

The SVM approximates the relation between input parameters $\mathbf{x} \in \mathbb{R}^{d_{L}}$ and class labels $y$ using a function of the form:

$$
g(\mathbf{x})=\mathbf{w}^{T} \boldsymbol{\varphi}(\mathbf{x})+b
$$

where $\varphi: \mathbb{R}^{d_{L}} \rightarrow \mathbb{R}^{d_{H}}$ is the feature map into a $d_{H}$ - dimensional feature space given a-priori, $\mathbf{w} \in \mathbb{R}^{d_{H}}$ the weight vector and $b \in \mathbb{R}$ the bias. Given a training set of $N$ pairs $\left(\mathbf{x}_{i}, y_{i}\right)$ the weight vector $\mathbf{w}$ and bias $b$ are chosen to solve the primal training problem:

$$
\begin{array}{ll}
\min _{\mathbf{w}, b} R(\mathbf{w}, b)=\frac{1}{2} \mathbf{w}^{T} \mathbf{w}+\frac{C}{N} \sum_{i \in \mathbb{Z}_{N}} \xi_{i} \\
\text { such that: } & \mathbf{w}^{T} \boldsymbol{\varphi}\left(\mathbf{x}_{i}\right)+b \leq y_{i}+\xi_{i} \forall i \in \mathbb{Z}_{N} \\
& \mathbf{w}^{T} \boldsymbol{\varphi}\left(\mathbf{x}_{i}\right)+b \geq y_{i}-\xi_{i} \forall i \in \mathbb{Z}_{N} \\
& \xi_{i} \geq 0 \forall i \in \mathbb{Z}_{N}
\end{array}
$$

The first term in the cost $R$ is a regularisation term included to minimise overfitting, while the second term is a measure of empirical risk (i.e. training set error). The parameter $C \in \mathbb{R}^{+}$controls the trade-off between risk minimisation and regularisation.

In practice, rather than solving the primal (2) directly the dual form of (2) is solved [15], namely:

$$
\begin{array}{cl}
\min _{\boldsymbol{\alpha}} Q(\mathbf{w}, b)= & \frac{1}{2} \sum_{i, j \in \mathbb{Z}_{N}} K_{i j} \alpha_{i} \alpha_{j}-\sum_{i \in \mathbb{Z}_{N}} \alpha_{i} \\
\text { such that: } & -\frac{C}{N} \leq \alpha_{i} \leq \frac{C}{N} \forall i \in \mathbb{Z}_{N} \\
& \sum_{i \in \mathbb{Z}_{N}} \alpha_{i} y_{i}=0
\end{array}
$$

where $K_{i j}=K\left(\mathbf{x}_{i}, \mathbf{x}_{j}\right)$ and $K(\mathbf{x}, \mathbf{y})=\varphi^{T}(\mathbf{x}) \boldsymbol{\varphi}(\mathbf{y})$ is the kernel function.

The trained machine may be written in terms of the dual variables as:

$$
g(\mathbf{x})=\operatorname{sgn}\left(\sum_{i \in \mathbb{Z}_{N}} \alpha_{i} K\left(\mathbf{x}_{i}, \mathbf{x}\right)+b\right)
$$

In the present study the inertial sensor signal features extracted at $M T C_{\text {Time }}$ were normalized to zero mean, unit variance (subtract the mean, divide by the SD) before testing with Support Vector Machine (SVM) to classify Young and Older gaits. Data from both young and older participants were randomized and one third of data was used to as the training set and the rest was used to test the model
Table 1: Mean and SD of inertial sensor kinematics obtained at $M T C_{\text {Time }}$ for Young and Older adults. * denotes significant difference between Young and Older adults

\begin{tabular}{|c|c|c|c|}
\hline $\begin{array}{c}\text { Inertial Sensor Feature } \\
\text { at } M T C_{\text {Time }}\end{array}$ & Young & Older & $\mathrm{p}$ \\
\hline AccX mean & -1.23 & -1.59 & 0.682 \\
AccY mean & 2.17 & 0.36 & $0.002^{*}$ \\
AccZ mean & -0.22 & -0.77 & 0.376 \\
GyroX mean & -324.94 & -297.62 & 0.150 \\
GyroY mean & 41.27 & 34.66 & 0.678 \\
GyroZ mean & 1.18 & -0.28 & 0.953 \\
AccX SD & 2.08 & 2.73 & $0.006^{*}$ \\
AccY SD & 1.07 & 1.92 & $0.005^{*}$ \\
AccZ SD & 1.69 & 2.15 & $0.014^{*}$ \\
GyroX SD & 14.60 & 23.39 & $10^{-3} *$ \\
GyroY SD & 20.17 & 20.12 & 0.984 \\
GyroZ SD & 24.56 & 28.09 & 0.168 \\
\hline
\end{tabular}

performance. Linear, and Gaussian RBF with $\mathrm{g}=1 \mathrm{e}-3$, $3 \mathrm{e}-3,1 \mathrm{e}-2, \ldots, 100,300)$ were examined. All the extracted inertial sensor features were used for classification and then only the inertial sensor signals which showed significant difference between the Young and the Older in the t-tests were used for classification.

1. Linear kernel: $K(\mathbf{x}, \mathbf{y})=\mathbf{x}^{T} \mathbf{y}$.

2. RBF kernel: $K(\mathbf{x}, \mathbf{y})=\exp \left(-\frac{1}{\sigma}\|\mathbf{x}-\mathbf{y}\|^{2}\right)$ (where $\left.\sigma \in \mathbb{R}^{+}\right)$.

\section{RESULTS}

Figure 3 shows time synchronized 3D motion capture vertical toe-displacement data and inertial sensor signals obtained from foot-mount sensor system. Table 1 presented mean and SD of 3 accelerometer features and 3 gyroscope features extracted at $M T C_{\text {Time }}$ for Young and Older separately. Only one mean was different between Young and Older, i.e., AccZ, sagittal planar vertical acceleration, was significantly greater in Young than Older. Standard deviations of all three acceleration signals and angular velocity about medio-lateral axis (GyroX) of Older were significantly greater than the Young (Figure 4). Other inertial sensor signals distributions characterised by mean and SD were not different between Young and Older.

When only the features that showed statistical differences were fed into the SVM classifiers, the best classification accuracy was obtained with linear kernel was $52.65 \%$ and with RBF 83.6\%. Gaussian RBF Kernel g was 0.9. When all six inertial sensor kinematics were used the accuracy improved to $92.1 \%$ with the RBF kernel.

\section{DISCUSSION}

The present study examined the inertial sensor kinematics obtained at $M T C_{\text {Time }}$ for both young and older. As older adults generally show greater variability in gait related biomechanical parameters such as stride-width, stride length, and most importantly MTC, it was expected that 

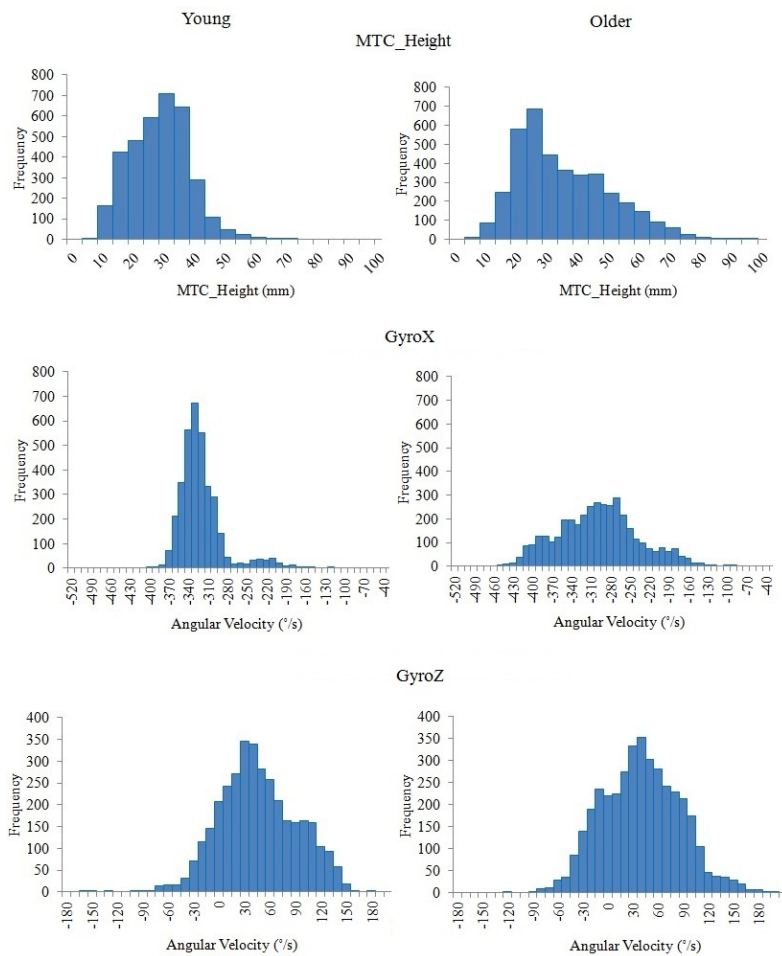

Figure 4: $M T C_{\text {Height }}$ distribution of Young and Older and a sample of distribution with (GyroX) and without (GyroZ) a significant different between the age groups

the inertial sensor kinematics obtained at $M T C_{\text {Time }}$ should also reflect such age-related biomechanical differences.

The study revealed that four of six inertial sensor kinematics obtained at critical toe-trajectory control event, MTC, were different between young and older, characterised either by mean or SD. Acceleration in the anterior-posterior axis $(\mathrm{Acc} Y)$ was significantly lower for Older than Young. The effect on anterior-posterior inertial kinematics could be possibly due to walking speed different between Young and Older. Older adults $(0.46 \mathrm{~m} / \mathrm{s})$ walked slower than the Young $(0.53$ $\mathrm{m} / \mathrm{s}$ ), however the difference in walking speed was not significantly different $(\mathrm{p}=0.07)$. The greater $\mathrm{AccY}$ at $M T C_{\text {Time }}$, therefore, was not only due to walking speed difference, but also possibly due to ageing effects. Variability of all three acceleration measurements obtained at $M T C_{\text {Time }}$ was significantly greater for Older than the Young. Further, variability of angular velocity about medio-lateral axis was also greater for Older. In consistent with literature, $M T C_{H e i g h t}$ distribution showed in Figure 4 also revealed greater variability in Older adults than Young. Greater variability in inertial sensor kinematics at $M T C_{\text {Time }}$ revealed reduced precision in low-limb trajectory control. The present study, therefore suggests that the three dimensional acceleration measurements and medio-lateral gyroscope reading at $M T C_{\text {Time }}$ could be used to differentiate older and young gaits. In the present study $M T C_{\text {Time }}$ was calculated using $3 \mathrm{D}$ motion capture to extracted kinematics at critical toe-trajectory control event, MTC. In future work, however, $M T C_{\text {Time }}$ could be obtained directly from intertial sensor kinematics by detecting the $2^{\text {nd }}$ peak (minimum) medio-lateral angular velocity, GyroX (Figure3). Further, in the future study, different classifiers such as Naive Bayes and logistical regression could be compared against SVM with cross-validation scheme.

The SVM classification with RBF kernel outperformed linear SVM classifier in differentiating young and older gaits using these inertial sensor kinematics obtained at $M T C_{\text {Time }}$ The SVM was, however, performing better when all six features were fed into the system than using only the features which showed statistically significant difference between young and older adults.

\section{CONCLUSION}

The present study showed that the inertial sensor kinematics obtained at $M T C_{\text {Time }}$ were characteristically different between young and older adults. Further the study demonstrated that these inertial sensor kinematics at $M T C_{\text {Time }}$ could be used to automatically classify young and older adults' gait with the aid of SVMs. These findings are significant in suggesting that the inertial sensors could be employed in clinical or research studies to differentiate older gaits in a non-laboratory setup while performing everyday tasks.

\section{REFERENCES}

[1] R. S. Barrett, P. M. Mills, and R. K. Begg. A systematic review of the effect of ageing and falls history on minimum foot clearance characteristics during level walking. Gait \&S Posture, 32(4):429-435, 2010.

[2] R. Begg, R. Best, L. Dell'Oro, and S. Taylor. Minimum foot clearance during walking: Strategies for the minimisation of trip-related falls. Gait \&5 Posture, 25(2):191-198, 2007. doi: 10.1016/j.gaitpost.2006.03.008

[3] N. Cristianini and J. Shawe-Taylor. An Introductino to Support Vector Machines and other Kernel-Based Learning Methods. Cambridge University Press, Cambridge, UK, 2005.

[4] R. Herbrich. Learning Kernel Classifiers: Theory and Algorithms. MIT Press, 2002.

[5] M. B. V. Iersel, R. P. C. Kessels, B. R. Bloem, A. L. M. Verbeek, and M. G. M. Olde Rikkert. Executive functions are associated with gait and balance in community-living elderly people. The Journals of Gerontology Series A: Biological Sciences and Medical Sciences, 63(12):1344-1349, 2008.

[6] D. T. H. Lai, R. Begg, and M. Palaniswami. Svm models for diagnosing balance problems using statistical features of the mtc signal. International Journal of Computational Intelligence $\&$ Applications, 7(3):317, 2008

[7] S. R. Lord and J. Dayhew. Visual risk factors for falls in older people. Journal of the American Geriatrics Society, 49(5):508-515, 2001.

[8] P. M. Mills, R. S. Barrett, and S. Morrison. Toe clearance variability during walking in young and elderly men. Gait \&s Posture, 28(1):101-107, 2008. doi: 10.1016/j.gaitpost.2007.10.006.

[9] W. H. Organization. Who global report on falls 
prevention in older age, 2007.

[10] M. J. Pavol, T. M. Owings, K. T. Foley, and M. D. Grabiner. Gait characteristics as risk factors for falling from trips induced in older adults. Journals of Gerontology - Series A Biological Sciences and Medical Sciences, 54(11):M583-M590, 1999.

[11] A. M. Sabatini, C. Martelloni, S. Scapellato, and F. Cavallo. Assessment of walking features from foot inertial sensing. Biomedical Engineering, IEEE Transactions on, 52(3):486-494, 2005.

[12] J. Shawe-Taylor and N. Cristianini. Kernel Methods for Pattern Analysis. Cambridge University Press, 2004.

[13] C. Sherrington, S. R. Lord, and C. F. Finch. Physical activity interventions to prevent falls among older people: update of the evidence. Journal of Science 8
Medicine in Sport, 7(1 Supplement):43-51, 2004.

[14] A. Smola. Regression estimation with support vector learning machines. Master's thesis, Technische Universität Münschen, 1996.

[15] A. Smola and B. Schölkopf. A tutorial on support vector regression. Technical Report NeuroCOLT2 Technical Report Series, NC2-TR-1998-030, Royal Holloway College, University of London, UK, October 1998.

[16] I. Steinwart and A. Christman. Support Vector Machines. Springer, 2008.

[17] V. Vapnik, S. Golowich, and A. Smola. Support vector methods for function approximation, regression estimation, and signal processing. In Advances in Neural Information Processing Systems, volume 9, pages 281-187. MIT Press, 1997. 